\title{
Confirmation of avian trichomonosis among wild birds in Ireland
}

\author{
Susan Doyle ${ }^{1} \cdot$ Margaret Reilly $^{1} \cdot$ Theo $^{\text {De Waal }}{ }^{2} \cdot$ Barry J. McMahon $^{1}$ (i)
}

Received: 11 December 2020 / Revised: 26 November 2021 / Accepted: 20 December 2021 / Published online: 19 January 2022 (c) The Author(s) 2022

\begin{abstract}
Trichomonas gallinae is the causative protozoan parasite of avian trichomonosis, an infectious disease of wild bird species. Since an outbreak in the UK in 2005, avian trichomonosis has resulted in mass mortality events in greenfinch (Chloris chloris) and chaffinch (Fringillia coelebs) populations. Given its proximity to the UK, it is important to monitor the emergence of $T$. gallinae in Ireland. This study proposed to sample greenfinch, goldfinch (Carduelis carduelis) and feral pigeon (Columba livia domestica) in Ireland with the aim of culturing and isolating $T$. gallinae. The oral cavity of birds was sampled with an oropharyngeal swab, which was then inoculated into Biomed InPouch ${ }^{\mathrm{TM}} \mathrm{TF}$ test kits. $T$. gallinae infection was diagnosed in 9 of 60 sampled birds ( 6 greenfinch and 3 feral pigeon), giving a total infection prevalence rate of $15 \%$ and providing evidence that avian trichomonosis is present among, but may not be limited to, greenfinch and feral pigeon populations in Ireland. It is also the first laboratory-based diagnosis of T. gallinae from live, free-living birds in Ireland to our knowledge. It is possible that avian trichomonosis is contributing to the recent decline in greenfinch populations in Ireland. Additionally, the presence of yeast cells in some positive samples did not appear to impede trichomonad growth, which may suggest a potential symbiotic relationship between $T$. gallinae and yeast species.
\end{abstract}

Keywords Avian parasitology $\cdot$ Emerging infectious diseases $\cdot$ Finches $\cdot$ Protozoal disease $\cdot$ Trichomonas gallinae

\section{Introduction}

Avian trichomonosis in wild birds is caused by the protozoan trichomonad Trichomonas gallinae (Amin et al. 2014). Trichomonosis is characterised by caseated, necrotic lesions of the respiratory and upper digestive tracts and may cause difficulty breathing and swallowing, leading to significant loss of weight and muscle mass and, ultimately, death (Hawn 1937; Amin et al. 2014). It has been linked to past population declines of several bird species including European turtle dove (Streptopelia turtur) and Mauritian pink pigeon (Nesoenas mayeri) (Bunbury et al. 2008) and is also believed to be one of the major contributing factors related to the extinction of passenger pigeon (Ectopistes migratorius) in North America (Stabler et al. 1954; Bunbury et al. 2007).

Barry J. McMahon

barry.mcmahon@ucd.ie

1 UCD School of Agriculture and Food Science, University College Dublin, Belfield, Dublin 4, Ireland

2 School of Veterinary Medicine, UCD, University College Dublin, Belfield, Dublin 4, Ireland
Trichomonosis is endemic to columbid (pigeon and dove) species, with feral pigeon (Columba livia domestica) considered to be the chief reservoir host (Stabler et al. 1954; Bunbury et al. 2007; Chi et al. 2013). It is highly transmissible among individuals (Stelter and Kummerfeld 2014); direct bill-to-bill transmission is particularly common, including from parent birds to nestlings (Amin et al. 2014). In recent years, trichomonosis has become an emerging infectious disease (EID) among fringillid (finch) species in Europe, most notably greenfinch (Chloris chloris). A dramatic decline in greenfinch abundance followed the emergence of trichomonosis in the UK; after an initial index case in 2005 , breeding populations of greenfinch declined by $35 \%$ (Robinson et al. 2010). Severe declines were observed in areas with high previously documented trichomonosis-related mortality, suggesting that a shift in T. gallinae host specificity to a novel species (greenfinch) had occurred.

It is likely that trichomonosis in finches may spread from the UK to neighbouring Ireland due to geographic proximity. Anecdotal evidence suggests an occurrence of trichomonosis among finches in Ireland; however, no official or definitive data exists. The Irish Garden Bird Survey (coordinated by BirdWatch Ireland; https://birdwatchireland.ie/) shows that 
current breeding greenfinch abundance is about half that recorded in the mid-2000s, coinciding with the emergence of trichomonosis in the UK. Thus, the objective of this study was to sample finch and pigeon populations in Ireland to test for the presence of $T$. gallinae. Our results provide the first laboratory-based diagnosis of $T$. gallinae from live, freeliving birds in Ireland.

\section{Materials and methods}

\section{Bird capture and sampling}

Three bird species (two fringillid and one columbid) were sampled for the presence of $T$. gallinae in Ireland: greenfinch, goldfinch (Carduelis carduelis) and feral pigeon. Greenfinch and goldfinch were selected due to their documented association with $T$. gallinae infection in the UK and mainland Europe (Amin et al. 2014; Chi et al. 2013; Robinson et al. 2010). Feral pigeon was selected to confirm the presence of $T$. gallinae, as this species is a known reservoir host (Stabler et al. 1954; Bunbury et al. 2007; Chi et al. 2013). A total of 20 individuals from each species were sampled, following Canon and Roe (1982) and to compromise between number of samples and logistical constraints. Finches were captured in a semi-rural area $\left(53^{\circ} 20^{\prime} \mathrm{N} 7^{\circ} 4^{\prime}\right.$ W) using mist nets on three occasions between 31 March and 15 April 2018. Feral pigeon were captured in an urban area $\left(53^{\circ} 20^{\prime} \mathrm{N} 6^{\circ} 15^{\prime} \mathrm{W}\right)$ using a hand net on four occasions between 25 April and 9 May 2018. The University College Dublin Animal Research Ethics Committee granted exemption from full ethical review for capture and sampling of these animals.

Upon capture, the species, sex, age, weight and the presence or absence of exterior beak and/or oropharyngeal lesions and discharge were recorded for each individual (Table 1). A salivary sample was collected using an oropharyngeal swab. Sterile, cotton-tipped swabs were gently inserted and rotated around the bird's oral cavity. The swab was then immediately placed inside an InPouch ${ }^{\mathrm{TM}} \mathrm{TF}$ Feline test kit (Biomed Diagnostics, White City, OR, USA) containing a growth medium to encourage trichomonad growth. Double-sampling of birds was avoided by marking finches with a metal British Trust of Ornithology issue leg ring and marking pigeons with ink on the tail feathers. After inoculation, the pouch was labelled and stored upright away from direct sunlight at an ambient temperature of approximately $20{ }^{\circ} \mathrm{C}$ until incubation.

\section{Culture and diagnosis}

On return from the field, inoculated pouches were incubated at $37{ }^{\circ} \mathrm{C}$ to allow for trichomonad growth. As the pouches were stored in an upright position, dense materials (including epithelial cells, debris, and, possibly, protozoal organisms) settled along the bottom chamber. This area of sediment was examined by light microscopy at magnification rates of $\times 100$ and $\times 400$ at $24-48$-h intervals using an Olympus System Microscope model BX40 for signs of protozoal activity, which is the gold standard method for diagnostic evaluation of protozoal infections in animals (De Waal 2012). Positive samples were removed from incubation when a diffuse amount of protozoal activity throughout the sediment was observed. Microscopic diagnosis of $T$. gallinae infection was confirmed based on the morphology of observed protozoa, i.e. four anterior flagella, an undulating membrane and axostyle (Tasca and De Carli 2003). If no protozoal activity or growth was observed after 14 days, samples were declared negative. In addition, the presence of yeast in any sample was recorded.

\section{Impact of infection on body condition}

The relationship between infection and body mass in greenfinch was tested using a linear regression model in $\mathrm{R}$ program (R Core Team 2021). Goldfinch and feral pigeon were excluded from this analysis because infection was not detected (goldfinch; see results) or the relevant biometrics were not recorded (feral pigeon; non-routine capture). Body mass was scaled for body size by dividing body weight by wing length, and sex and age were included as blocking variables. We also included an interaction term between infection status and the presence of yeast in the sample to test whether the presence of yeasts influences the severity of the impact of infection on body condition.

\section{Results}

Positive cases of T. gallinae were observed in 9 of the 60 pouches analysed (Table 2). Six greenfinch pouches displayed signs of trichomonad growth and contained a thick, opaque sediment layer, with the presence of what appeared to be yeast. Signs of trichomonad activity became evident between $24 \mathrm{~h}$ and 11 days incubation (Fig. 1). Three feral pigeon pouches also displayed signs of trichomonad growth and contained a thick, opaque sediment layer. The presence of yeast in this instance was clear due to the build-up of gas inside several pouches as a result of fermentation. One pouch displayed signs of trichomonad activity even before incubation commenced, while the other two pouches displayed signs between 4 and 7 days incubation. No sign of trichomonad activity was observed in any of the goldfinch pouches. A small sediment layer was observed in each pouch, most likely consisting of epithelial cells and residual debris from the oral cavity. 
Table 1 For every sampled bird, the species, age (adult or juvenile), sex, biometrics (weight in grams, wing in millimetres), notes on body condition and $T$. gallinae infection result (positive or negative) was recorded. An "NA" indicates that measurements were not/could not be taken for the individual

\begin{tabular}{|c|c|c|c|c|c|c|c|}
\hline Sample & Species & Age & Sex & Weight & Wing & Condition & Infection \\
\hline 1 & Goldfinch & Juvenile & Unknown & 14.3 & 76 & No remarks & Negative \\
\hline 2 & Goldfinch & Juvenile & Male & 15.9 & 79 & No remarks & Negative \\
\hline 3 & Goldfinch & Juvenile & Female & 27.1 & 85 & No remarks & Negative \\
\hline 4 & Goldfinch & Adult & Female & 15.8 & 79 & No remarks & Negative \\
\hline 5 & Goldfinch & Juvenile & Female & 15.2 & 77 & No remarks & Negative \\
\hline 6 & Goldfinch & Adult & Female & 16.9 & 78 & No remarks & Negative \\
\hline 7 & Greenfinch & Juvenile & Male & 22.4 & 86 & Mucous & Positive \\
\hline 8 & Goldfinch & Juvenile & Male & 16.5 & 79 & No remarks & Negative \\
\hline 9 & Greenfinch & Adult & Male & 26.8 & 88 & No remarks & Negative \\
\hline 10 & Goldfinch & Juvenile & Male & 15.1 & 78 & No remarks & Negative \\
\hline 11 & Goldfinch & Juvenile & Male & 13.9 & 79 & No remarks & Negative \\
\hline 12 & Goldfinch & Adult & Female & 14.0 & 75 & No remarks & Negative \\
\hline 13 & Greenfinch & Juvenile & Male & 24.9 & 87 & Oral discharge & Positive \\
\hline 14 & Goldfinch & Juvenile & Female & 13.5 & 74 & No remarks & Negative \\
\hline 15 & Goldfinch & Juvenile & Female & 14.3 & 78 & No remarks & Negative \\
\hline 16 & Goldfinch & Juvenile & Female & 15.1 & 77 & No remarks & Negative \\
\hline 17 & Goldfinch & Unknown & Unknown & 16.8 & 79 & No remarks & Negative \\
\hline 18 & Goldfinch & Juvenile & Male & NA & 75 & No remarks & Negative \\
\hline 19 & Greenfinch & Juvenile & Male & 25.8 & 89 & No remarks & Negative \\
\hline 20 & Goldfinch & Adult & Unknown & 14.0 & 76 & No remarks & Negative \\
\hline 21 & Goldfinch & Juvenile & Female & 13.6 & 73 & No remarks & Negative \\
\hline 22 & Goldfinch & Juvenile & Female & 15.8 & 76 & No remarks & Negative \\
\hline 23 & Goldfinch & Juvenile & Male & 15.0 & 74 & No remarks & Negative \\
\hline 24 & Goldfinch & Juvenile & Male & 14.7 & 77 & No remarks & Negative \\
\hline 25 & Greenfinch & Juvenile & Female & 26.8 & 84 & No remarks & Negative \\
\hline 26 & Goldfinch & Juvenile & Female & 16.0 & 78 & No remarks & Negative \\
\hline 27 & Greenfinch & Juvenile & Female & 21.7 & 85 & Mucous & Positive \\
\hline 28 & Greenfinch & Juvenile & Male & 26.6 & 85 & No remarks & Negative \\
\hline 29 & Greenfinch & Juvenile & Male & 28.8 & 85 & No remarks & Negative \\
\hline 30 & Greenfinch & Adult & Male & 23.8 & 86 & No remarks & Positive \\
\hline 31 & Greenfinch & Juvenile & Male & 25.2 & 86 & No remarks & Negative \\
\hline 32 & Greenfinch & Juvenile & Male & 25.9 & 88 & No remarks & Positive \\
\hline 33 & Greenfinch & Adult & Female & 26.6 & 82 & Oral discharge & Negative \\
\hline 34 & Greenfinch & Juvenile & Male & 26.3 & 87 & No remarks & Negative \\
\hline 35 & Greenfinch & Juvenile & Male & 28.8 & 90 & No remarks & Negative \\
\hline 36 & Greenfinch & Juvenile & Male & 24.2 & 87 & Oral discharge & Positive \\
\hline 37 & Greenfinch & Juvenile & Female & 25.3 & 88 & No remarks & Negative \\
\hline 38 & Greenfinch & Adult & Male & 25.2 & 87 & No remarks & Negative \\
\hline 39 & Greenfinch & Adult & Male & 26.1 & 92 & No remarks & Negative \\
\hline 40 & Greenfinch & Juvenile & Female & 25.1 & 82 & No remarks & Negative \\
\hline 41 & Pigeon & Unknown & Unknown & 350 & NA & No remarks & Negative \\
\hline 42 & Pigeon & Unknown & Unknown & 330 & NA & No remarks & Negative \\
\hline 43 & Pigeon & Unknown & Male & 340 & NA & No remarks & Negative \\
\hline 44 & Pigeon & Unknown & Male & 410 & NA & No remarks & Positive \\
\hline 45 & Pigeon & Unknown & Unknown & NA & NA & No remarks & Negative \\
\hline 46 & Pigeon & Unknown & Male & 325 & NA & No remarks & Negative \\
\hline 47 & Pigeon & Unknown & Male & 345 & NA & No remarks & Negative \\
\hline 48 & Pigeon & Unknown & Female & 330 & NA & No remarks & Negative \\
\hline 49 & Pigeon & Unknown & Unknown & 300 & NA & No remarks & Positive \\
\hline 50 & Pigeon & Unknown & Unknown & 375 & NA & No remarks & Negative \\
\hline 51 & Pigeon & Unknown & Unknown & 335 & NA & No remarks & Negative \\
\hline
\end{tabular}


Table 1 (continued)

\begin{tabular}{llllllll}
\hline Sample & Species & Age & Sex & Weight & Wing & Condition & Infection \\
\hline 52 & Pigeon & Unknown & Male & 365 & NA & No remarks & Negative \\
53 & Pigeon & Unknown & Male & 395 & NA & No remarks & Negative \\
54 & Pigeon & Unknown & Male & 355 & NA & Oral discharge & Negative \\
55 & Pigeon & Unknown & Unknown & 315 & NA & No remarks & Negative \\
56 & Pigeon & Unknown & Unknown & 325 & NA & No remarks & Negative \\
57 & Pigeon & Unknown & Male & 360 & NA & No remarks & Negative \\
58 & Pigeon & Unknown & Unknown & 250 & NA & No remarks & Positive \\
59 & Pigeon & Unknown & Unknown & 330 & NA & No remarks & Negative \\
60 & Pigeon & Unknown & Unknown & 345 & NA & No remarks & Negative \\
\hline
\end{tabular}

Our linear regression model detected a relationship between body mass and infection in greenfinch (Fig. 2). Scaled body mass was on average $11 \%$ lower in infected birds, after accounting for sex and age, and this relationship was significant $(P=0.002)$. This model explained $39 \%$ of variation in body mass, but we acknowledge that the sample size was low $(n=20)$; therefore, this result must be considered with caution. Testing body weight (as opposed to mass) revealed similar results: after accounting for sex and age, the body weight of infected greenfinch was on average $2.9 \mathrm{~g}$ less than uninfected greenfinch (average uninfected weight of $25.5 \mathrm{~g}$ ), explaining $47 \%$ of variation in body weight. Our model did not detect a synergetic effect of yeast and infection on body condition, or an additive effect of yeast.

\section{Discussion}

This study confirms that the protozoal organism $T$. gallinae is present among greenfinch and feral pigeon populations in Ireland and may be considered an EID of greenfinch in an Irish setting. It is also the first laboratory-based diagnosis of T. gallinae from live, free-living birds in Ireland to our knowledge. Despite the small sample size (20 individuals per species) and the small geographical area covered, $T$. gallinae was readily diagnosed. Infection prevalence in

Table 2 The total number of samples, positive cases and incubation time (hours or days for each sample) are presented for the three study species

\begin{tabular}{llll}
\hline Species & Samples & $\begin{array}{l}\text { Positive } \\
\text { cases }\end{array}$ & Incubation time \\
\hline Greenfinch & 20 & 6 & $24 \mathrm{~h}: n=2$ \\
& & $48 \mathrm{~h}: n=1$ \\
& & $72 \mathrm{~h}: n=2$ \\
& & 11 days: $n=1$ \\
Feral pigeon & 20 & 3 & $<24$ h: $n=1$ \\
& & & 4 days: $n=1$ \\
Goldfinch & 20 & 0 & - \\
\hline
\end{tabular}

greenfinch, feral pigeon and goldfinch was 30\%, 15\% and 0\% respectively, with a disease prevalence of the entire sample set being $15 \%$.

It has been widely suggested that bird-feeders facilitate the spread of infection among birds, especially common garden birds such as greenfinch and goldfinch (Lawson et al. 2012). During the study, we noted that not all birds with positive infection cases exhibited visible signs of infection during capture. However, four positively tested greenfinch had foamy, slightly sanguineous salivary discharge or excessive mucoid discharge. During early trichomonad growth stages in the pouches, the motility of the active trichomonads generally appeared as small areas of movement in the sediment layer of the pouch. In later stages, movement became more diffuse throughout the sediment layer, as trichomonads proliferated. Live, motile trichomonads were observed utilising their anterior flagella under the microscope. Specimens ranged from spherical and ovoidal to piriform in shape, and the axostyle was also observed.

The presence of $T$. gallinae in Ireland may have significant consequences for finch population trends. Our results suggest that greenfinch are much more susceptible to $T$. gallinae infection than goldfinch. However, although T. gallinae infection was absent from our goldfinch samples, Robinson et al. (2010) confirmed that goldfinch is affected by trichomonosis in the UK, albeit to a much lesser extent than greenfinch. Avian trichomonosis is known to have negative population level effects on species abundance (e.g. Robinson et al. 2010) and may already be impacting finch abundance in Ireland. We found that infected greenfinch had, on average, an $11 \%$ lower body mass than non-infected individuals, suggesting a significant negative impact of infection on body condition (noting that the sample size in this test was 20 birds). Therefore, it may be the case that trichomonosis is negatively impacting greenfinch, especially when considered in tandem with the observed decline of breeding greenfinch evident in Irish Garden Bird Survey Data, but has not yet impacted goldfinch populations as an EID in Ireland.

This variation in susceptibility could be related to yeast; in our samples, high yeast burdens did not appear to impede 
Fig. 1 Actively dividing trichomonads observed at $\times 1000$ magnification. Note the presence of 2 nuclei in each cell

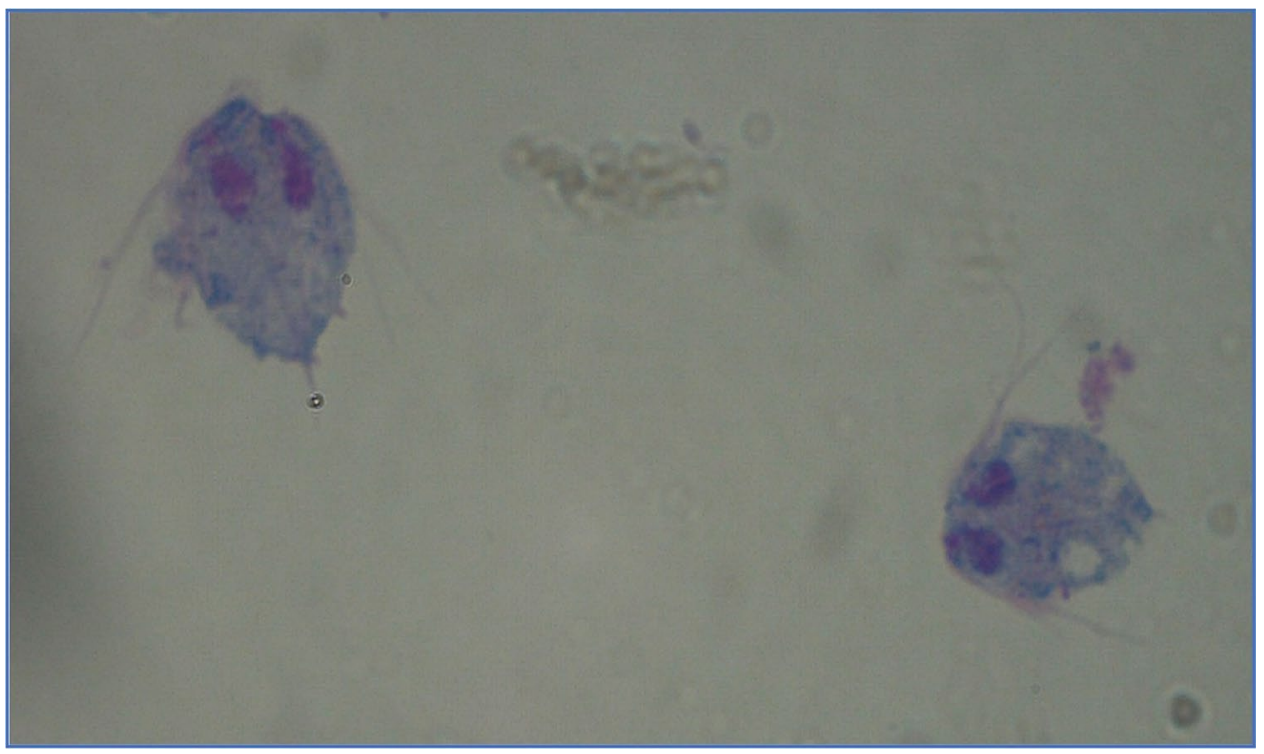

trichomonad proliferation. Furthermore, co-infection of trichomonads and yeast has been observed previously (e.g. co-infection of Trichomonas vaginalis and Candida spp. in humans; Avila et al. 2017; Brown et al. 2004). Our analysis did not detect an influence of yeasts on the severity of the impact of infection on body condition. However, the potential relevance of yeast to variation in the emergence of $T$. $\mathrm{gal}$ linae among species warrants further investigation. Further
Fig. 2 Relationship between body mass and $T$. gallinae infection status in greenfinch. Here, body mass was scaled for body size by dividing bird weight $(\mathrm{g})$ by wing length (mm). The data show that birds with no infection tended to have a significantly higher body mass

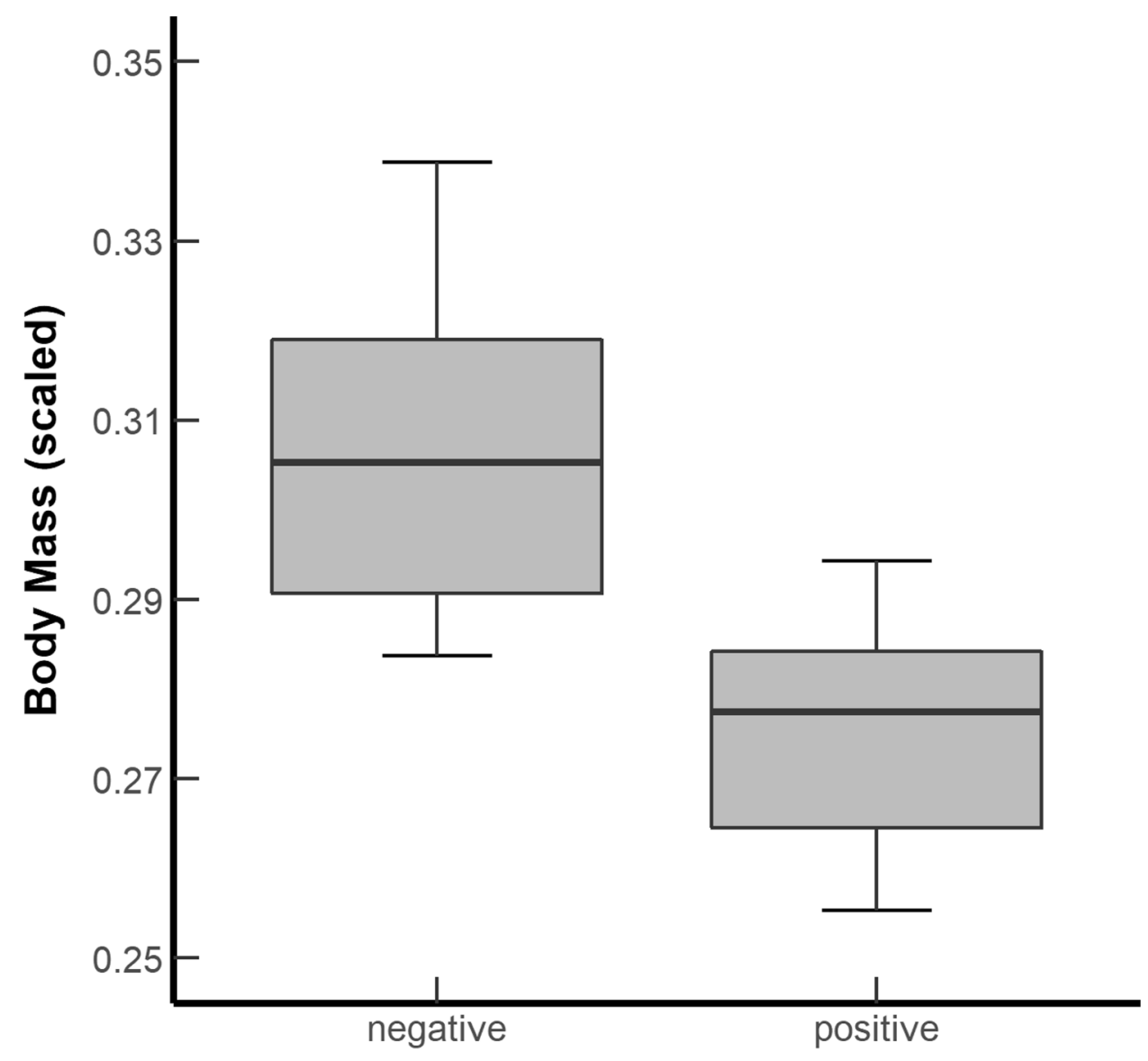

Infection Status 
sampling of a wider variety of species over a greater geographic range will also provide a more accurate picture of the spread and prevalence of avian trichomonosis as an EID in Ireland.

Acknowledgements This research derives from M.Sc. studies in Wildlife Conservation and Management at University College Dublin. The authors wish to thank Declan Manley and Patrick Manley for their advice and assistance in the capture and handling of the birds that were sampled for this study.

Funding Open Access funding provided by the IReL Consortium.

Open Access This article is licensed under a Creative Commons Attribution 4.0 International License, which permits use, sharing, adaptation, distribution and reproduction in any medium or format, as long as you give appropriate credit to the original author(s) and the source, provide a link to the Creative Commons licence, and indicate if changes were made. The images or other third party material in this article are included in the article's Creative Commons licence, unless indicated otherwise in a credit line to the material. If material is not included in the article's Creative Commons licence and your intended use is not permitted by statutory regulation or exceeds the permitted use, you will need to obtain permission directly from the copyright holder. To view a copy of this licence, visit http://creativecommons.org/licenses/by/4.0/.

\section{References}

Amin A, Bilic I, Hess LD, M, (2014) Trichomonads in birds-a review. Parasitology 141:733-747

Avila JAC, Garcia MLS, Parra JF, Puerto AS, Mari JMN, Stensvold CR, Fernandez JG (2017) Prevalence and genetic diversity of Trichomonas vaginalis in the general population of Grenada and co-infections with Gardnerella vaginalis and Candida species. J Med Microbiol 66:1436-1442
Brown HL, Fuller DD, Jasper LT, Davis TE, Wright JD (2004) Clinical evaluation of affirm VPIII in the detection and identification of Trichomonas vaginalis, Gardnerella vaginalis, and Candida Species in Vaginitis/Vaginosis. Infect Dis Obstet Gynecol 12:17-21

Bunbury N, Jones CG, Greenwood AG, Bell DJ (2007) Trichomonas gallinae in Mauritian columbids: implications for an endangered endemic. J Wildl Dis 43:399-440

Bunbury N, Jones CG, Greenwood AG, Bell DJ (2008) Epidemiology and conservation implications of Trichomonas gallinae infection in the endangered Mauritian pink pigeon. Biol Cons 141:153-161

Canon RM, Roe RT (1982). Livestock disease surveys: a field manual for veterinarians. Australian Bureau of Animal Health

Chi JF, Lawson B, Durrant C, Beckmann K, John S, Alrefaei AF, Kirkbride K, Bell DJ, Cunningham AA, Tyler KM (2013) The finch epidemic strain of Trichomonas gallinae is predominant in British non-passerines. Parasitology 140:1234-1245

De Waal T (2012) Advances in diagnosis of protozoan diseases. Vet Parasitol 189:65-74

Hawn MC (1937) Trichomoniasis of turkeys. J Infect Dis 61:184-197

Lawson B, Robinson RA, Colvile KM, Peck KM, Chantrey J, Pennycott TW, Simpson VR, Toms MP, Cunningham AA (2012) The emergence and spread of finch trichomonosis in the British Isles. Philos Trans R Soc B 367:2852-2863

R Core Team (2021) R: a language and environment for statistical computing. R Foundation for Statistical Computing, Vienna, Austria

Robinson RA, Lawson B, Toms MP, Peck KM, Kirkwood JK, Chantrey J, Clatworthy IR, Evans AD, Hughes LA, Hutchinson OC and John SK (2010) Emerging infectious disease leads to rapid population declines of common British birds. PLoS One 5:e12215

Stabler RM (1954) Trichomonas gallinae: a review. Exp Parasitol 3:368-402

Stelter R, Kummerfeld N (2014) Avian trichomonosis. Tierarztliche. Umschau 69:133-140

Tasca T, De Carli GA (2003) Scanning electron microscopy study of Trichomonas gallinae. Vet Parasitol 118:37-42

Publisher's Note Springer Nature remains neutral with regard to jurisdictional claims in published maps and institutional affiliations. 